\title{
REDES DE PESQUISA E INOVAÇÃO: UM ESTUDO DAS RELAÇÕES DA PETROBRAS COM INSTITUIÇÕES DE CIÊNCIA E TECNOLOGIA DO ESTADO DE SÃO PAULO
}

\author{
Alessandro Augusto Jordão (alessandrojordao@unip.br) - Instituto de Ciências Exatas e Tecnologia \\ (ICET), Universidade Paulista (UNIP); Departamento de Economia (DEc-So), Universidade Federal \\ de São Carlos (UFSCar). \\ Patrícia Saltorato (patrisal@ dep.ufscar.br) - Programa de Pós-Graduação em Engenharia de Produção \\ (PPGEPS), Universidade Federal de São Carlos (UFSCar).
}

Cleyton Fernandes Ferrarini (cleyton@ufscar.br) - Departamento de Engenharia de Produção (DEPS), Universidade Federal de São Carlos (UFSCar).

\section{RESUMO}

A intensificação das relações academia-indústria se revela uma estratégia para o fortalecimento dos sistemas de inovação. Por esta razão, tem havido no país um aumento expressivo de discussões na academia e no âmbito das políticas públicas sobre os mecanismos institucionais mais adequados para estimular a interação entre a academia e as indústrias. No entanto, a criação de mecanismos institucionais impõe uma co-evolução às instituições de pesquisa e ensino superior e aos setores industriais que deve ser gerenciada com cuidado, de modo que possa garantir resultados tecnológicos no curto prazo, sem comprometer as trajetórias de desenvolvimento do conhecimento no longo prazo. Apoiada pela abordagem evolucionista e neo-shumpeteriana do pensamento econômico heterodoxo, $e$ por uma abordagem histórica da co-evolução técnica e institucional do sistema de inovação da indústria petrolífera brasileira, esta pesquisa buscou explorar as relações da Petrobras com instituições de ciência e tecnologia do Estado de São Paulo por intermédio das suas redes temáticas de pesquisa. Apoiada em um estudo exploratório, e de caráter descritivo e analítico, esta pesquisa revelou que, ao articular atores com capacitações inovativas distintas (e complementares), através das redes temáticas de pesquisa, a Petrobras tem reforçado seu acervo tecnológico, e ao mesmo tempo, oportunizado às instituições de ciência e tecnologia do Estado de São Paulo a produção de conhecimento em áreas científicas de fronteira. Além do mais, ao fomentar, através das redes temáticas de pesquisa, a criação de infra-estrutura permanente e específica de pesquisa, a requalificação de infra-estrutura de pesquisa existente e a qualificação de recursos humanos especializados, a Petrobras tem ampliado a capacidade de pesquisa, desenvolvimento e inovação $(P, D \& I)$ de diferentes grupos de pesquisa das instituições de ciência e tecnologia do Estado de São Paulo, qualificando-os para o atendimento de demandas tecnológicas regionais.

Palavras-chave: Relação Universidade-Empresa; Petrobras; Rede de Pesquisa; Inovação.

Área: Organização e gestão do conhecimento para o desenvolvimento de produtos 


\section{INTRODUÇÃOO}

O No Brasil, a história do sistema de inovação da indústria petrolífera está fortemente interligada a evolução da Petrobras, empresa estatal criada no início da década de 1950 (MORAIS, 2012; FUCK et al, 2007; FURTADO, 2004).

Até a década de 1990, período que antecede a quebra do monopólio estatal, a Petrobras monopolizava as atividades de exploração, produção, refino e transporte de petróleo e seus derivados. Além disso, dominava exclusivamente as atividades de financiamento, coordenação, execução e uso de novas tecnologias (FURTADO, 2004). Até então, o sistema de inovação da indústria petrolífera nacional era dominado por um único ator: a Petrobras.

No entanto, com a promulgação da Lei do Petróleo (n ${ }^{\circ}$ 9478), em 1997, a Petrobras deixa de ter o monopólio legal, e são incorporados novos atores institucionais ao sistema de inovação da indústria petrolífera nacional, tais como: o Conselho Nacional de Política Energética (CNPE) e a Agência Nacional do Petróleo, atual Agência Nacional de Petróleo, Gás e Bicombustíveis (ANP).

A Petrobras, que antes, dominava os vértices de políticas públicas, regulação e operação, a partir da promulgação de Lei do Petróleo, fica restrita ao vértice de operação, deixando ao Conselho Nacional de Política Energética (CNPE) e à Agência Nacional do Petróleo (ANP) os vértices de ordem pública e de regulação, respectivamente (BONNELI E VEIGA, 2003).

Como mencionado, a Lei do Petróleo (Lei n ${ }^{\circ}$ 9478/1997) instituiu a Agência Nacional de Petróleo (ANP), e estabeleceu dentre suas atribuições, a competência de estimular a pesquisa e o desenvolvimento de tecnologias inovadoras nas atividades de exploração, produção, refino e processamento de petróleo e gás natural (TORRES E COSTA, 2013).

Como resultado desta determinação, em 1998, a ANP incluiu nos contratos de concessão para exploração, desenvolvimento e produção de petróleo e gás natural uma Cláusula de Investimento em Pesquisa e Desenvolvimento (Cláusula 24/ANP), exigindo das concessionárias com participação especial dispêndios compulsórios em atividades de pesquisa, desenvolvimento e inovação $(P, D \& I)$ em parceria com instituições de pesquisa e ensino superior do país.

O montante de recursos provenientes da Cláusula de Investimento em Pesquisa e Desenvolvimento acumulado no período de 2002 a 2012 foi de aproximadamente 7,2 bilhões de reais, e a Petrobras foi a concessionária com maior participação, respondendo por cerca de 93\% dos recursos arrecadados (ANP, 2013).

Para gerenciar tais recursos, a Petrobras criou, em 2006, as redes temáticas de pesquisa, um modelo de gestão compartilhada de pesquisa, desenvolvimento e inovação (P,D\&I) com instituições de pesquisa e ensino superior do país (POLETTO et al., 2011).

De acordo com o Centro de Pesquisa e Desenvolvimento "Leopoldo Américo Miguez de Mello" (Cenpes) da Petrobras, no período de 2006 a 2012, foram alocados 2,6 bilhões de reais em instituições de pesquisa e ensino superior do país, sendo destinado à região sudeste uma parcela de $71 \%$. Do total destinado à região sudeste (1,8 bilhões de reais), $24,9 \%$ foi investido nas instituições de pesquisa e ensino superior do Estado de São Paulo.

Assim, a Cláusula de Investimento em Pesquisa e Desenvolvimento, e as mudanças que ela acarretou, contribuiu para uma alteração no sistema de inovação da indústria petrolífera 
brasileira, diminuindo o centralismo exercido pela Petrobras, e aumentando a participação de outros atores, como as instituições de pesquisa e de ensino superior do país.

Essa alteração institucional impõe uma co-evolução às instituições de pesquisa e ensino superior e às empresas do setor petrolífero nacional que deve ser gerenciada com cuidado, de modo que possa garantir resultados tecnológicos no curto e médio prazo, sem comprometer as trajetórias de desenvolvimento do conhecimento no longo prazo.

A presente pesquisa teve o intuito de explorar as relações da Petrobras com as instituições de ciência e tecnologia do Estado de São Paulo. A proposição norteadora da pesquisa é que ao interagir com instituições de ciência e tecnologia do Estado de São Paulo, por intermédio das redes temáticas de pesquisa, a Petrobras tem reforçado seu acervo tecnológico e oportunizado externalidades científicas e sociais para a economia e a sociedade.

\subsection{Procedimentos metodológicos}

Para alcançar o objetivo proposto e constatar a proposição da pesquisa, foi realizado um estudo exploratório, com abordagem descritiva, utilizando-se métodos qualitativos de levantamento e coleta de informações.

O levantamento de informações se deu inicialmente junto à Petrobras, através do Centro de Pesquisa e Desenvolvimento "Leopoldo Américo Miguez de Mello" (Cenpes), e junto ao Diretório de Grupos de Pesquisa do Conselho Nacional de Pesquisa (CNPq). Isso permitiu a identificação das parcerias da Petrobras com as Instituições de Ciência e Tecnologia do Estado de São Paulo, bem como dos gestores institucionais e dos pesquisadores acadêmicos envolvidos com as redes temáticas de pesquisa.

Para evitar distorções ligadas a uma eventual relação específica da Petrobras com uma determinada instituição, a pesquisa seguiu um caráter multi-institucional, e explorou as relações da Petrobras com cinco diferentes instituições de ciência e tecnologia do Estado de São Paulo, sendo estas: o Instituto de Pesquisas Tecnológicas do Estado de São Paulo (IPTSP), o Centro Nacional de Pesquisa em Energia e Materiais (CNPEM), a Universidade Federal de São Carlos (UFSCar), a Universidade Estadual de Campinas (UNICAMP) e a Universidade de São Paulo (USP).

\section{COORDENAÇÃO E GESTÃo DAS REDES TEMÁTICAS DE PESQUISA}

A presente seção trata de descrever a estrutura organizacional das redes temáticas de pesquisa investigadas e reportar a percepção dos respondentes quanto às práticas de coordenação e gestão empregadas.

A coexistência de diferentes modalidades de coordenação é uma característica própria de arranjos interinstitucionais de pesquisa (CALLON, 1991).

Essa característica foi observada nas redes temáticas de pesquisa investigadas, visto que, nestas redes a coordenação é compartilhada por diferentes atores institucionais: o Cenpes/Petrobras e as instituições de ciência e tecnologia.

Apesar de existir uma dominância do Cenpes/Petrobras na rede, os atores institucionais lideram conjuntamente diversas iniciativas científico-tecnológicas relacionadas à condução dos projetos de pesquisa, e se alternaram nas atividades de gestão da rede, o que conforma uma estrutura de coordenação híbrida. 
No que se refere à estrutura organizacional das redes temáticas de pesquisa investigadas, foi tentativamente proposto um padrão de governança comum.

O controle das atividades técnico-científicas desenvolvidas no âmbito de um projeto temáticos de pesquisa é realizado por um coordenador de pesquisa específico. Esse coordenador é encarregado também da gestão técnica-administrativa do projeto de pesquisa.

É importante ressaltar que uma rede temática de pesquisa agrupa diferentes projetos temáticos de pesquisa, e cada projeto de pesquisa é coordenado por um pesquisador líder, que não pertence necessariamente à mesma ICTs dos outros coordenadores.

As atividades técnico-científicas desenvolvidas no âmbito de uma rede são acompanhadas e avaliadas por um comitê técnico-científico que, em geral, é composto por pesquisadores do Cenpes/Petrobras e de diferentes ICTs. De acordo com os respondentes, o comitê técnicocientífico assume um papel importante, principalmente no que se refere a tomadas de decisões para ações futuras da rede temática de pesquisa.

A coordenação executiva e operacional da rede é realizada por um gestor técnico do Cenpes/Petrobras, que confere e faz diligências sobre as atividades técnico-científicas e também sobre a atividade contábil e financeira dos projetos de pesquisa. É importante ressaltar que essas últimas ficam a cargo dos coordenadores de pesquisa e, em alguns casos, a participação das fundações de apoio institucional das ICTs foi expressiva.

De acordo com respondentes, o papel do gestor na rede temática é essencial, visto que, este realiza a interlocução entre os coordenadores de pesquisa de diferentes projetos temáticos com o comitê técnico-científico da rede.

Na Figura 1, pode ser visualizado um esquema genérico da estrutura de governança das redes temáticas investigadas. É importante ressaltar que as redes temáticas de pesquisa não assumem uma estrutura organizacional pré-defina e que, em alguns casos, podem divergir quanto a algumas práticas.

Figura 1 - Estrutura de Governança das Redes Temáticas

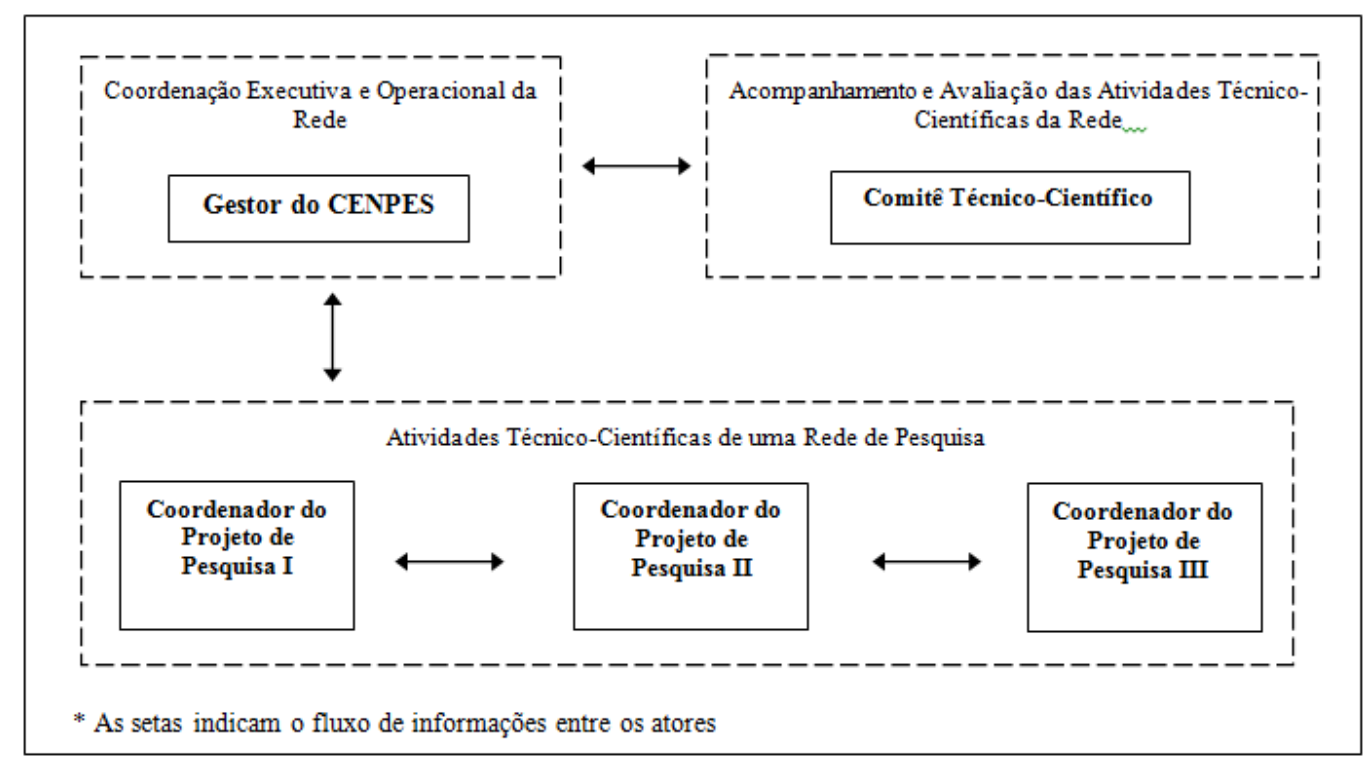

Fonte: JORDÃO (2014).

Quanto à conformação das redes temáticas de pesquisa investigadas, foi observado que estas se estabeleceram através de relações formalizadas, envolvendo contratos e convênios de 
cooperação que definem o teor das atividades científicas e tecnológicas que são desenvolvidas, bem como a disponibilização e apropriação dos resultados gerados.

De acordo com os respondentes, as relações contratuais ofereceram segurança ao processo, mas, em alguns casos, envolveram uma burocracia excessiva, dificultando a realização das parcerias e à continuidade dos projetos temáticos de pesquisa.

Em relação à estrutura das redes temáticas de pesquisa investigadas, foi possível identificar que, ao mesmo tempo em há flexibilidade de comunicação e interação entre os atores, há um relacionamento hierárquico associado a questões gerenciais, à prestação de contas e à demonstração de resultados.

Quanto à percepção dos respondentes sobre as práticas administrativas e de gestão das redes temáticas de pesquisa, os respondentes destacaram que o sucesso do projeto temático de pesquisa está fortemente associado ao sucesso nas atividades de liderança, tendo em vista a importância do papel do gestor e dos coordenadores de pesquisa no estabelecimento de um contexto favorável à aquisição e transmissão de conhecimentos e informações.

De acordo com os respondentes, os projetos de pesquisa foram acompanhados continuamente por seus respectivos gestores tanto de forma presencial quanto por meios virtuais, tais como: correio eletrônico, telefonemas e teleconferências.

Em alguns casos, foram solicitados aos coordenadores de pesquisa relatórios técnicos sumarizados além dos relatórios previstos no plano de trabalho. Em outros casos, foram agendadas reuniões presenciais ou "tele presenciais" para a apresentação dos resultados parciais e finais.

Os respondentes ressaltaram também que a realização de reuniões técnico-científicas e workshops gerais com a presença de pesquisadores de outras redes de pesquisa era uma oportunidade efetiva de grande difusão de informações e conhecimentos, principalmente porque eram pensadas em conjunto formas de melhorar o andamento dos projetos temáticos de pesquisa, entretanto estas reuniões não estão sendo mais realizadas com a regularidade de antes.

É importante ressaltar a contribuição das tecnologias de informação e comunicação para a coordenação das atividades em rede, visto que, nos casos estudados, estas potencializaram a interação interpessoal, levando a um intercambio de experiências e a uma troca de conhecimentos mais efetiva entre os atores das redes investigadas.

\section{IMPACTOS CIENTÍFICOS, TECNOLÓGICOS E SOCIAIS}

A presente seção reporta os resultados científicos, tecnológicos e não tecnológicos alcançados pelas redes de pesquisa investigadas, e analisa a percepção de diferentes atores sobre esses resultados.

$\mathrm{Na}$ percepção dos respondentes, a interação da Petrobras com os grupos de pesquisa investigados tem possibilitado a produção de conhecimentos de fronteira, e tem resultado na nacionalização de áreas científicas que, anteriormente, eram dominadas exclusivamente por instituições de pesquisa estrangeiras. Foi observado que a interação em rede criou um ambiente propício ao desenvolvimento cientifico, e tem ampliado as competências científicas e tecnológicas dos agentes envolvidos.

Considerando o fato de que $46 \%$ dos projetos de pesquisa das redes investigadas se encontram em um estágio intermediário de desenvolvimento e $15 \%$ em um estágio embrionário, temos que, a produção científica e tecnológica das redes de pesquisa investigadas foi significativa. 
De acordo com os respondentes, a produção científica está relacionada principalmente à realização de pesquisas em nível de graduação e pós-graduação, à publicação de artigos em periódicos nacionais e internacionais e à elaboração de relatórios e notas técnicas.

Os respondentes sinalizaram que o conteúdo científico produzido a partir das redes de pesquisa investigadas tem alcançado reconhecimento em circuitos acadêmicos internacionais, corroborando para um aumento da visibilidade internacional das instituições de ciência e tecnologia envolvidas, e oportunizando o estabelecimento de parcerias internacionais ligadas a pesquisas de outra natureza.

Alguns pesquisadores acadêmicos, em especial, relataram que o potencial de produção científica das redes de pesquisa é maior, mas encontra-se limitado em decorrência de cláusulas de confidencialidade impostas pelo Cenpes/Petrobras.

É importante ressaltar também que, em alguns casos, houve uma colaboração profícua entre pesquisadores acadêmicos e pesquisadores e técnicos da Petrobras que resultou no desenvolvimento de trabalhos e instrumentos científicos. Essa informação revela a ocorrência de relações pontuais de retroalimentação entre a dimensão científica e tecnológica, tal como indicava a revisão bibliográfica.

Com base nas informações disponibilizadas pelos respondentes, temos que, o potencial de produção científica das redes de pesquisa investigadas é elevado, e que o conteúdo científico resultante foi bastante significativo, contradizendo os autores que afirmam que a relação academia-indústria compromete a produção científica dos pesquisadores acadêmicos.

Em relação à produção tecnológica das redes de pesquisa investigadas, temos que, $35 \%$ dos respondentes indicaram ter patentes depositadas no Instituto Nacional de Propriedade Industrial (INPI), 15\% indicaram ter patentes depositadas em escritórios de patentes internacionais, tais como: United States Patent and Trademark Office (USPTO), o World Intellectual Property Organization (WIPO) e o European Patent Office (EPO), e 18\% indicaram ter patentes concedidas por escritórios de patentes nacionais e internacionais. Além disso, foram relatados resultados consideráveis no que se refere à produção de novos produtos, processos e protótipos tecnológicos.

$\mathrm{Na}$ percepção de alguns respondentes, o potencial de inovação das redes de pesquisa é elevado, e a geração de patentes só não foi mais expressiva porque muitos projetos de pesquisa se encontram em estágio intermediário de desenvolvimento. Esses respondentes, em especial, atribuíram às redes de pesquisa um potencial de inovação elevado por conta de investimentos acentuados na criação de infra-estrutura de pesquisa e na modernização da infra-estrutura de pesquisa disponível.

Como apontado na imagem a seguir, $43 \%$ das redes de pesquisa investigadas envolvem projetos de pesquisa com objetivos estratégicos e de longo prazo. Diante disso, é importante ressaltar que as redes de pesquisa investigadas iniciaram suas atividades a partir do ano de 2006. E por esta razão, muitos projetos se encontram em um estágio intermediário de desenvolvimento e não alcançaram ainda seus objetivos esperados. Soma-se, ainda, o fato de que a concessão de uma patente envolve um processo técnico-administrativo complexo, cuja tramitação pode levar um tempo considerável. Ao considerar essas observações, a perspectiva é que a geração de patentes seja mais acentuada futuramente. 
Figura 2 - Impactos Tecnológicos Alcançados pelas Redes de Pesquisa Investigadas

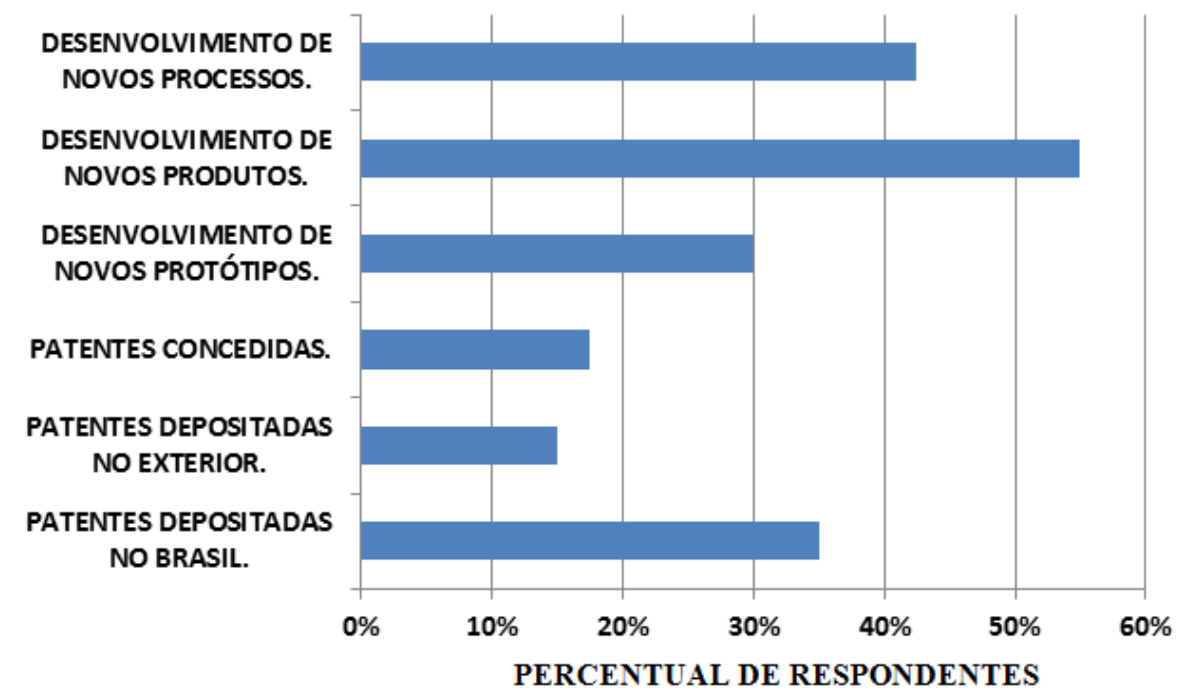

Fonte: JORDÃO (2014).

Além dos resultados científicos e tecnológicos gerados pelas redes de pesquisa investigadas, foram relatados resultados relacionados à criação de infra-estrutura permanente e específica de pesquisa, à requalificação de infra-estrutura de pesquisa existente, e à formação e qualificação de recursos humanos.

Na percepção dos respondentes, a criação e a melhoria da infra-estrutura de pesquisa ampliou a capacidade de pesquisa do grupo de pesquisadores, qualificando-os para o atendimento de demandas tanto do setor petrolífero e para-petrolífero quanto de outros setores industriais, que podem se beneficiar da capacidade laboratorial e do conhecimento específico criado.

Em muitos casos, a infra-estrutura laboratorial construída ou melhorada oportunizou a captação de outros recursos provenientes da Fundação de Apoio à Pesquisa do Estado de São Paulo (FAPESP) e de agências de fomento federais, tais como: CAPES, CNPq e FINEP.

Quanto à formação e qualificação de recursos humanos, alguns pesquisadores revelaram que as redes de pesquisa oportunizaram melhoramentos no ensino de graduação e pós-graduação, e a inserção de estudantes em pesquisas de nível internacional. Em decorrência disso, os estudantes tem se inserido com mais facilidade no mercado de trabalho.

Ainda sobre a questão de formação e qualificação de recursos humanos, os respondentes relataram que a formação de competências especializadas tem alcançado também os profissionais da Petrobras, visto o envolvimento desses com programas de pós-graduação das instituições de ciência e tecnologia investigadas.

\section{CONSIDERAÇÕES FINAIS}

Apoiada pela abordagem evolucionista e neo-shumpeteriana do pensamento econômico heterodoxo, e por uma abordagem histórica da co-evolução técnica e institucional do sistema de inovação da indústria petrolífera brasileira, esta pesquisa buscou explorar as relações da Petrobras com as instituições de ciência e tecnologia do Estado de São Paulo por intermédio das suas redes temáticas de pesquisa. 
Através de um estudo exploratório, e de caráter descritivo e analítico, a pesquisa explorou, mais especificamente: o conteúdo científico, tecnológico e não tecnológico resultante da interação.

Ao integrar atores com capacitações inovativas distintas (e complementares), nomeadamente os técnicos e pesquisadores do Cenpes/Petrobras e os pesquisadores das instituições de ciência e tecnologia, as redes temáticas de pesquisa tem oportunizado externalidades científicas e tecnológicas para os atores envolvidos, e externalidades sociais para a economia e a sociedade.

As redes temáticas de pesquisa investigadas têm gerado um conteúdo tecnológico relativamente considerável, relacionado, em síntese, ao desenvolvimento de produtos, processos e protótipos tecnologicamente melhorados ou inovadores, resultando em patentes depositadas em escritórios de propriedade intelectual e industrial - nacionais e internacionais.

Contradizendo a idéia de que a relação academia-indústria atende exclusivamente ao interesse das indústrias, e compromete a produção científica da academia, as redes temáticas de pesquisa investigadas apresentaram resultados científicos relativamente significativos que, em síntese, estão relacionados à realização de pesquisas em nível de graduação e pós-graduação, e a consequiente publicação de artigos em periódicos nacionais e internacionais.

Ao fomentar a produção de conhecimentos de fronteira nas instituições de ciência e tecnologia do Estado de São Paulo, as redes temáticas de pesquisa investigadas tem oportunizado a nacionalização de áreas científicas que, anteriormente, eram dominadas exclusivamente por instituições de pesquisa estrangeiras.

Além de estarem contribuindo para o fortalecimento do sistema setorial de inovação da indústria petrolífera brasileira, as redes temáticas de pesquisa investigadas tem oportunizado o desenvolvimento científico e tecnológico regional.

Buscando viabilizar as atividades de pesquisa, desenvolvimento e inovação (P,D\&I) das redes temáticas de pesquisa, a Petrobras investiu recursos consideráveis na criação e requalificação da infra-estrutura laboratorial e de pesquisa nas instituições de ciência e tecnologia do Estado de São Paulo.

Ao fomentar a criação de infra-estrutura permanente e específica de pesquisa e a requalificação de infra-estrutura de pesquisa existente, tem-se ampliado a capacidade de pesquisa, desenvolvimento e inovação (P,D\&I) dos grupos de pesquisa envolvidos, qualificando-os para o atendimento de demandas tecnológicas regionais.

É importante ressaltar que a infra-estrutura laboratorial construída ou melhorada tem oportunizado às instituições de ciência e tecnologia envolvidas a captação de outros recursos provenientes da Fundação de Amparo à Pesquisa do Estado de São Paulo (FAPESP) e de agências de fomento federais, tais como: CAPES, CNPq e FINEP, para o desenvolvimento de outras atividades de P,D\&I, que não da Petrobras.

Através da inserção de jovens pesquisadores (graduandos e pós-graduandos) em pesquisas de "fronteira", as redes temáticas de pesquisa têm oportunizado melhoramentos no ensino de graduação e pós-graduação. E, em decorrência disso, os estudantes tem se inserido com mais facilidade no mercado de trabalho.

Como tratado anteriormente, a Petrobras, induzida por um mecanismo institucional setorial, qual seja, a Cláusula de Dispêndio obrigatório em Pesquisa e Desenvolvimento (P\&D), criou as redes temáticas de pesquisa, um modelo de interação academia-indústria, que, como mostra essa pesquisa, tem contribuído com o acervo científico-tecnológico da Petrobras, sem comprometer, no caso, as atividades acadêmicas das instituições de ciência e tecnologia do 
Estado de São Paulo que, na realidade, ao interagirem com a Petrobras, ampliaram suas capacitações científicas e tecnológicas.

\section{REFERÊNCIAS}

BONNELI, R.; VEIGA, P. M. A dinâmica das Políticas Setoriais no Brasil na década de 1990: Continuidade e Mudança. Revista Brasileira de Comércio Exterior, n. 75, 2003.

ETZKOWITZ, H. Hélice Tríplice: universidade-indústria-governo: inovação em ação. Porto Alegre: EDIPUCRS, 2009.

FIORAVANTE, D.; AGUIRRE, L. A cooperação entre universidades e empresas e os fornecedores da PETROBRAS. In: TURCHI, L.; NEGRI, F.; NEGRI, J. A. Impactos Tecnológicos das Parcerias da PETROBRAS com Universidades, Centros de Pesquisa e Firmas Brasileiras. $1^{a}$ edição. Brasília: IPEA, 2013. cap. 4, p. 115-138.

FUCK, M. P.; RIBEIRO, C. G.; BONACELLI, M. B. M.; FURTADO, A. T. P\&D de interesse público? Observações a partir do estudo da EMBRAPA e da PETROBRAS. Engevista, v. 9, n. 2, p. 85-99, Dez. 2010.

FURTADO, A. T. Mudança Institucional e Inovação na Indústria Brasileira de Petróleo. In: KON, A.; OLIVEIRA, G. Pesquisa Industrial Trabalho e Tecnologia, , Fapesp, 2004.

FURTADO, A. T.; PEREIRA, N. M.; MARZANI, B. S. Política de compras da Petrobras: a nova relação contratual. X Congresso Brasileiro de Energia, p. 1729-1744, 2004.

JORDÃO, ALESSANDRO AUGUSTO. A influência das relações academia-indústria no desenvolvimento tecnológico: um estudo das relações da Petrobras com instituições de ciência e tecnologia do Estado de São Paulo. Ano de 2014. Dissertação (Mestrado em Engenharia de Produção) - Centro de Ciências e Tecnologias para Sustentabilidade, Universidade Federal de São Carlos, Sorocaba, 2014.

LA ROVERE, R. L.; RODRIGUES, R. F. Outsourcing and Diffusion of Knowledge in ICT Clusters: A Case Study. In: PYKA, A.; FONSECA, M. G. D. Catching up, spillovers and Innovation Networks in a Schumpeterian Perspective. Berlin: Springer-Verlag, 2011.

MONTOBBIO, F.; STERZI, V. Inventing Together: Exploring the Nature of International Knowledge Spillovers in Latin America. In: PYKA, A.; FONSECA, M. G. D. Catching up, spillovers and Innovation Networks in a Schumpeterian Perspective. Berlin, 2011. cap 4, p 81-118.

MORAIS. J. M. Petróleo em águas profundas: Uma história tecnológica da Petrobras na exploração e produção offshore. Brasília: IPEA, 2012, 423 p.

PELLEGRIN, I.; NUNES, M.; ANTUNES JÚNIOR, J. Impacto tecnológico dos projetos desenvolvidos pela PETROBRAS em parceria com instituições de ensino e pesquisa da região sul do Brasil. In: TURCHI, L.; NEGRI, F.; NEGRI, J. A. Impactos Tecnológicos das Parcerias da PETROBRAS com Universidades, Centros de Pesquisa e Firmas Brasileiras. $1^{a}$ edição. Brasília: IPEA, 2013. cap. 09, p. 267-320.

POLETTO, C. A.; ARAÚJO, M. A. D.; MATA, W. Gestão compartilhada de P\&D: o caso da Petrobras e a UFRN. Revista de Administração Pública, v. 45, n. 4, p. 1095-1117, ago 2011. 
ROCHA, A. M. S.; ROMERO, F. Technology Evaluation and Licensing in Portuguese Universities. In: INTERNATIONAL CONFERENCE ON APPLIED BUSINESS AND ECONOMICS. 2010, Coruña.

SALERNO, M. S.; FREITAS, S. L. T. U. A influência da PETROBRAS no desenvolvimento tecnológico: O caso dos institutos de ciência e tecnologia na região sudeste. In: TURCHI, L.; NEGRI, F.; NEGRI, J. A. Impactos Tecnológicos das Parcerias da PETROBRAS com Universidades, Centros de Pesquisa e Firmas Brasileiras. $1^{a}$ edição. Brasília: IPEA, 2013. cap. 10, p. 321-358.

SUZIGAN, W.; ALBUQUERQUE, E. A interação entre universidades e empresas em perspectiva histórica no Brasil. In SUZIGAN, W; ALBUQUERQUE, E.; CAIRO, S. Em busca da inovação: interação universidade-empresa no Brasil. Belo Horizonte, Autêntica Editora, 2011. p. 17-44.

TORRES, E.; COSTA, J. Modelo de Gestão das Redes Temáticas e Núcleos Regionais da Petrobras/ANP. Revista Capacite. Editora UFS. São Cristovão, 2013. 